\title{
Image segmentation of Bullet appearance defects based on color model and hybrid threshold
}

\author{
Wang peng, Guo Chao-yong, Liu Hong-ning \\ Ordnance Engineering College. Hebei Shijiazhuang 050003 China
}

Keywords: image segmentation; color model; OTSU algorithm; two-peak algorithm

\begin{abstract}
Aiming at the question of defect image segmentation in quality detection of bullet appearance. Through the analysis of color characteristics of bullet appearance defects and combined with OTSU algorithm and two-peak algorithm, Proposes a hybrid threshold segmentation method based on color model. The method first extracts component map R , using OTSU algorithm to segmentation high light part of the image, and finally with two-peak algorithm to the further defect segmentation. Application of Matlab simulation results show that, this method can be successfully applied to the bullet appearance defect segmentation.
\end{abstract}

\section{General Introduction}

The bullet is the most important part of light weapons, whose role it is self-evident. In order to ensure the quality in the production process of bullets, they must to detect bullet surface, to eliminate defective cartridge. At present, China's military enterprises generally adopt manual work to detect the bullets surface, and the manual sorting way after found the defective bullets. This traditional testing method has low efficiency, low accuracy and high labor intensity, reduced the degree of automation of the production of bullet. With the development of science and technology progressing, and the bullet's quality and production efficiency requirements increasing, while the artificial detection method of cartridge surface have been unable to meet the requirements of the automatic production of the era, how to improve the quality, efficiency and automation degree of the bullet appearance inspection, is a very important problems to be solved. Automatic detection method for image processing can achieve defect, improve the efficiency of the detection.

In the automatic detection of defects, defect image segmentation is a key for defect identification, the segmentation quality directly affects the subsequent image processing effects, and even determine the success or failure. Because the surface of a metal cylinder bullet is smooth surface, the appearance of the image acquisition process cartridges often occur in high light phenomenon, which makes it unable to complete the bullet appearance defect segmentation by gray level image, while color images provide more information than the gray image, so get more and more attention. In order to accurately, rapidly segment image defect, from the analysis of the color features of defects and color feature comparison bullets body appearance, the method of using RGB color model and hybrid threshold segmentation to determine the defect area of the bullet appearance.

\section{Gray threshold segmentation of the defect}

\subsection{OTSU algorithm}

There are many kinds of appearance defects of bullets, discuss several bullet appearance defects of the most common here, including green spot, indentation and dirt. As shown in fig 1. 


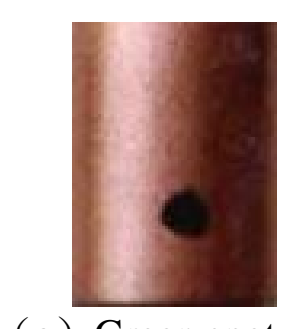

(a) Green spot
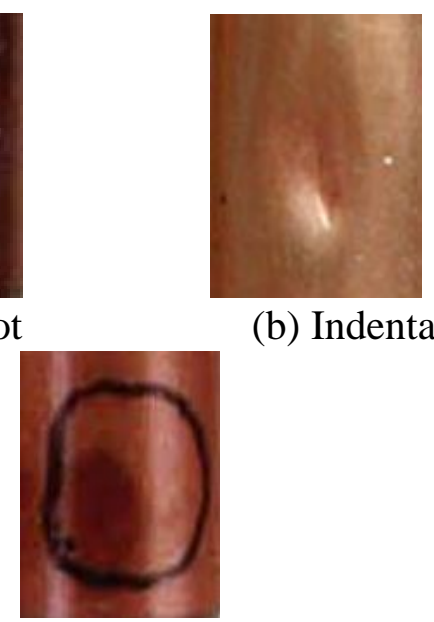

(b) Indentation

(c)Dirt

Fig:1 Appearance defects of bullets

The most basic gray threshold segmentation method is two-peak algorithm and OTSU algorithm, OTSU algorithm was proposed in 1979 by Otsu in Japan, the segmentation of image is calculated as follow.

The pixels in a digital image is divided into $C_{0}$ and $C_{1}$ types according to the gray level in $f(x, y)$ with the threshold, i.e.

$$
\left\{\begin{array}{l}
C_{0}=\left\{f_{1}(x, y) \mid f_{\text {min }} \leq f(x, y) \leq T\right\} \\
C_{1}=\left\{f_{2}(x, y) \mid f_{\text {max }} \geq f(x, y) \geq T\right\}
\end{array}\right.
$$

Among them, $f_{\min } 、 f_{\max }$ were gray minimum and maximum value in the image. Let $N_{i}$ be the number of pixel whos gray value is $i\left(f_{\min } \leq i \leq f_{\max }\right)$, then the total pixel image is $N=\sum_{i} N_{i}$,therefore, the each probability of the gray levels for $P(i)=N_{i} / N$, then the probability of $C_{0}$ is $\quad P_{0}=\sum_{i=f_{\min }}^{T} P(i)$

The mean is $u_{0}=\sum_{i=f_{\min }}^{T} i P(i) / P_{0}$

The probability of $C_{1}$ is $P_{1}=\sum_{i=T+1}^{f_{\max }} P(i)$

The mean is $\quad u_{1}=\sum_{i=T}^{f_{\max }} i P(i) / P_{1}$

The mean of whole $f(x, y)$ is $u=P_{0} u_{0}+P_{1} u_{1}$

Then, the definition of the class variance between the two types is $\sigma^{2}(T)=P_{0}\left(u-u_{0}\right)^{2}+P_{1}\left(u-u_{1}\right)^{2}$

The best threshold $T^{*}$ is that When $\sigma^{2}(T)$ is at maximum, namely to meet

$$
T^{*}=\left\{T^{*} \mid \sigma^{2}\left(T^{*}\right) \geq \sigma^{2}(T), \forall T \in\left[f_{\min }, f_{\max }\right]\right\}
$$

Use the OTSU method to segmented the three images in Fig 1 , as shown in fig 2 . The segmentation threshold were green spot is 111 , indentation is 105 , dirt 58 . We can see from Fig 2,due to the high light, OTSU algorithm cannot successfully segment bullet defect image. 

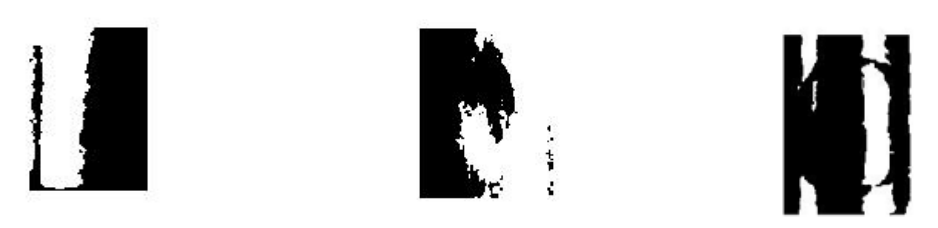

（a）Green spot $\mathrm{T}=111$ (b) Indentation $\mathrm{T}=105$ （c） Dirt $\mathrm{T}=58$

Fig:2 OTSU algorithm threshold segmentation

1.2 two-peak algorithm

Two-peak algorithm law that gray distribution curve of the image can be approximated by two normal distribution function $\left(u_{1}, \sigma_{1}^{2}\right)$ and $\left(u_{2}, \sigma_{2}^{2}\right)$.Two superimposed image will appear in two-peak distribution, as shown in Fig 3.

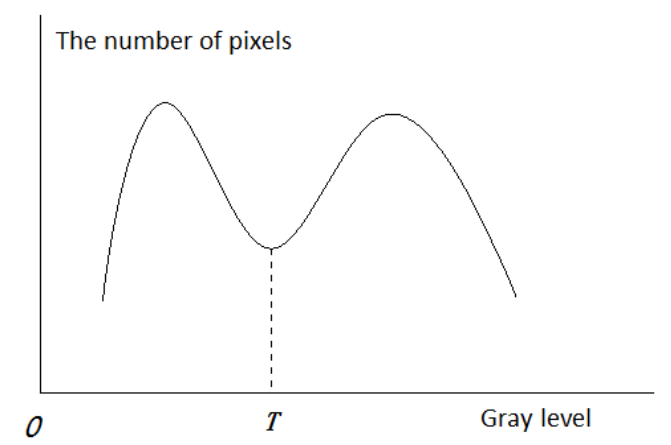

Fig:3 two-peak algorithm

Selection at the trough histogram, gray level $T$, image can be divided into two parts, the segmentation results of two-peak algorithm can be expressed as

$$
g(x, y)= \begin{cases}0 & f(x, y)<T \\ 1 & f(x, y) \geq T\end{cases}
$$

Use the two-peak algorithm to segmented the three images in Fig 1, as shown in fig 4. The segmentation thresholdwere green spot is 75 , indentation is 110 , dirt 64 . We can see from Fig4
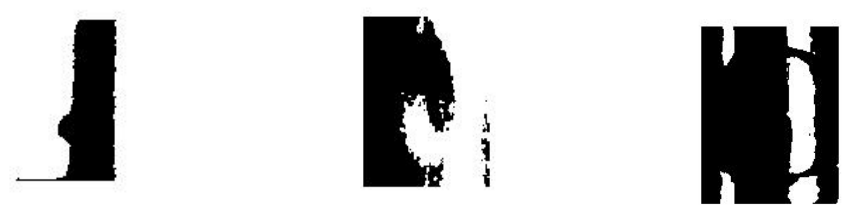

(a) Green spot $\mathrm{T}=75$ (b) Indentation $\mathrm{T}=110 \quad$ （c） Dirt $\mathrm{T}=64$

Fig:4 Two-peak algorithm threshold segmentation

\section{Color features of Defect}

Commonly used color model is RGB color model and HIS color model. In this paper, the RGB color model is used to analyze the image.

Taking the green spot for example, randomly select, highlight image part, normal part and the defect part 100 pixel to analysis. Extract the RGB curve, the results as shown in figure 5. 


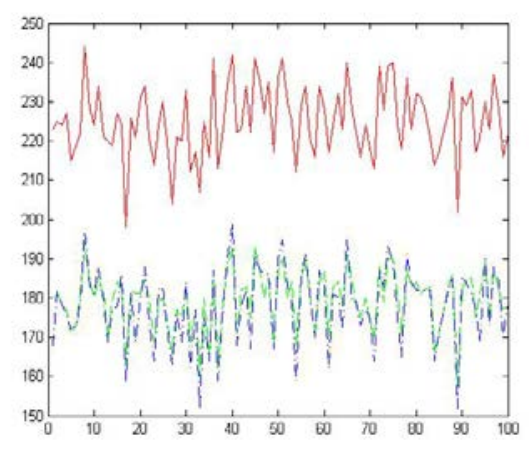

(a) highlight image part

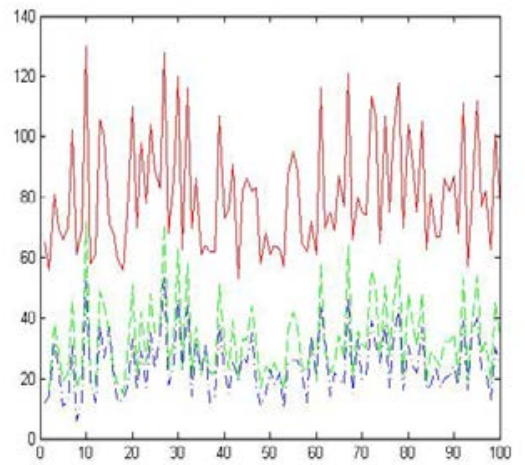

(b) normal part

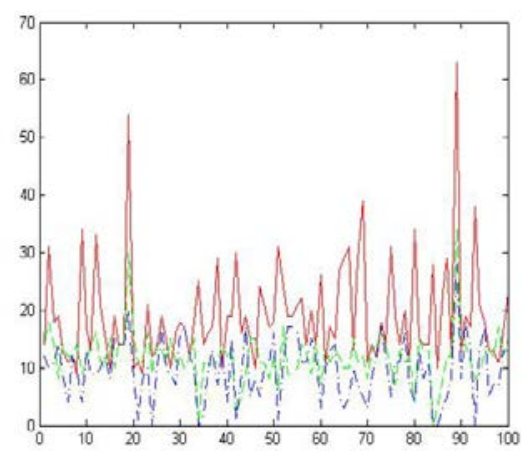

(c) defect part

Fig:5 RGB curve

The three part shows the comparison of three images are $R>G>B$. The $R$ component in the three part level obviously respectively high light $190<R<250$, normal part of $57<R<140$, the defect part is $0<\mathrm{R}<65$. Due to the defect of $\mathrm{R}$ component is larger than the pixel 65 accounted for a few, think the defect part $0<\mathrm{R}<55$. So will eliminate the overlapping part of the normal part of the $\mathrm{R}$ component and the R component of the defective part. The observation of Gcomponent and $\mathrm{B}$ component size difference is not obvious, and in the normalpart and defective part range overlap part of the larger, in order to better segmentation of the defect image, here we choose the $\mathrm{R}$ component as the main study, Figure 6 (b) on R component of the image segmentation by OTSU.
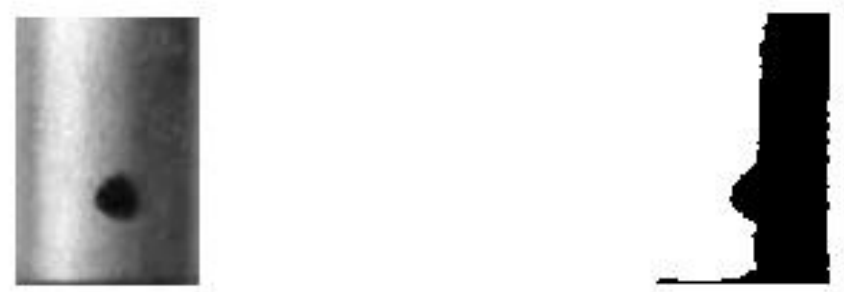

(a) Green spot R component (b) segmentation by OTSU

Fig:6 R component and OTSU segmentation

As can be seen, only the image of the R component of the OTSU method for asegmentation cannot fully segmented defect, so the gray image after segmentation value is 0 part with the component map $\mathrm{R}$ instead, see Figure 7(a) image shown, and then use the average gray of R component diagraminstead of part of the filled gray value of 0 parts, such as Figure 7 (b) image shown. 


\section{(a) R component instead （b ) after filled \\ Fig:7 R component instead and filled}

At this point, the image histogram as shown in Figure 8 (a), according to the gray level histogram, application of two-peak algorithm that $\mathrm{T}=41$,and anti get figure 8 (b) image shown.

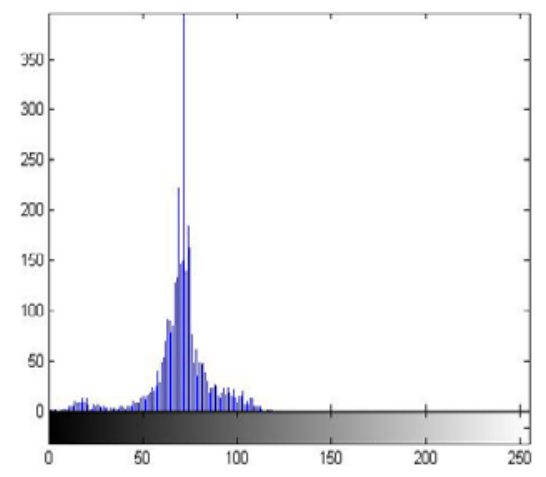

(a) gray level histogram

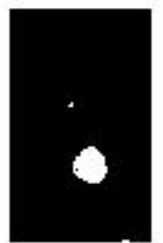

(b) two-peak algorithm segmentation

Fig:8 Further segmentation

Post processing of morphology, first of all to figure 8 (b) for corrosion, and then expansion, eliminate the micro area, such as shown in Figure 9 (a)results. Figure 9 (b) and Figure 9 (c) respectively using this method on the indentation and dirt processing results.

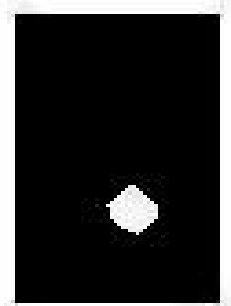

(a) Green spot

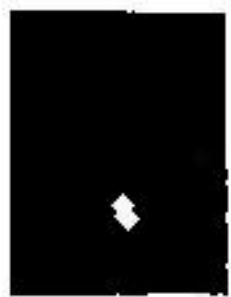

(b) Indentation

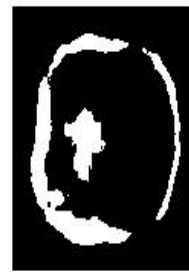

(c) Dirt

Fig:9 The final image processing 


\section{Conclusion}

This paper start from gray level segmentation method of bullets appearance defect. The applicability of the OTSU and two-peak algorithm is discussed, because the OTSU and twopeak algorithm unable to successful segment bullet appearance defects. On the basis of this, proposed a method that based on RGB model and OTSU combine with two-peak algorithm. First, extract the $\mathrm{R}$ component of the image, use OTSU to segment $\mathrm{R}$ component diagram, the image of the high light separation part will be separated from the image, and then use the method of two-peak algorithm successfully segment defects region. Finally, using this method respectively to the indentation and dirt defects segmentation, the results show that this method can successfully segment green spot, indentation and dirt defects.

\section{References}

[1] Liu gang, Wang lixiang Dong yan. MATLAB Digital image processing. Beijing:Mechanical industry press. 2010.

[2] Lin kaiyan, Wu junhui, Xu lihong. A survey on color image segmentation techniques.Jouenal of Image Graphics. 10(1):1-10. 2005.

[3] Jianbo Shi, Jitendra Malik. Normalized cuts and image segmentation[J]. IEEE Transactions on pattern analysis and machine intelligence.22(8):888-902.2000.

[4] Jiang linlin, Yu fenghua, Yue shida. Research satus on improved mehods of color image segmentation. Modern ecological agriculture. 3,4:95-101.2013.

[5] Otsu N.A threshould selection method from gray-level histograms[J].IEEE Transactions on System.Man and Cybernetics. 9(1):62-66.1979.

[6] Shi jinwei,Guo chaoyong,Liu hongning. Study on system of bullet surface defect based on machine vision.Modular Machine Tool\&Automatic Manufacturing Technique . (4):59-64.2013.

[7] Zhang yajun,Qi xinglin,Dong chengji.Automated test system for bullet steel core based on machine vision. Ordnance Industry Automation.33(6):59-61. 2012. 